\title{
Control of Basal Ganglia Output by Direct and Indirect Pathway Projection Neurons
}

\author{
Benjamin S. Freeze, ${ }^{1,2,3}$ Alexxai V. Kravitz, ${ }^{1}$ Nora Hammack, ${ }^{1}$ Joshua D. Berke, ${ }^{4}$ and Anatol C. Kreitzer ${ }^{1,2,3,5}$ \\ ${ }^{1}$ Gladstone Institute of Neurological Disease, San Francisco, California 94158, ${ }^{2}$ Biomedical Sciences Program and ${ }^{3}$ Medical Scientist Training Program, \\ University of California, San Francisco, California 94117-1049, ${ }^{4}$ Department of Psychology, and Neuroscience Program, University of Michigan, Ann Arbor, \\ Michigan 48109-1340, and 5Departments of Physiology and Neurology, University of California, San Francisco, California 94117-1049
}

The direct and indirect efferent pathways from striatum ultimately reconverge to influence basal ganglia output nuclei, which in turn regulate behavior via thalamocortical and brainstem motor circuits. However, the distinct contributions of these two efferent pathways in shaping basal ganglia output are not well understood. We investigated these processes using selective optogenetic control of the direct and indirect pathways, in combination with single-unit recording in the basal ganglia output nucleus substantia nigra pars reticulata $(\mathrm{SNr})$ in mice. Optogenetic activation of striatal direct and indirect pathway projection neurons produced diverse cellular responses in SNr neurons, with stimulation of each pathway eliciting both excitations and inhibitions. Despite this response heterogeneity, the effectiveness of direct pathway stimulation in producing movement initiation correlated selectively with the subpopulation of inhibited $\mathrm{SNr}$ neurons. In contrast, effective indirect pathway-mediated motor suppression was most strongly influenced by excited SNr neurons. Our results support the theory that key basal ganglia output neurons serve as an inhibitory gate over motor output that can be opened or closed by striatal direct and indirect pathways, respectively.

\section{Introduction}

Basal ganglia research over the past 20 years has been guided by a model (Albin et al., 1989; DeLong, 1990) in which the direct and indirect pathway projections from striatum achieve approximately opposite behavioral effects, via opposing effects on basal ganglia output nuclei. The direct pathway is thought to inhibit firing of GABAergic basal ganglia output nuclei such as substantia nigra pars reticulata $(\mathrm{SNr})$, thereby disinhibiting thalamocortical and brainstem circuitry and promoting movement. In contrast, the indirect pathway is hypothesized to increase $\mathrm{SNr}$ firing by subthalamic nucleus (STN)-mediated excitation, thus inhibiting downstream circuitry and suppressing movement. This model has had considerable heuristic value, but the validity of some basic assumptions has not been tested in behaving animals.

The clearest indication of SNr function in motor behavior comes from work on oculomotor control in nonhuman primates (Hikosaka and Wurtz, 1983). SNr was found to strongly inhibit the superior colliculus (SC) via a monosynaptic pathway, with pauses in SNr activity enabling SC neurons to generate saccades.

\footnotetext{
Received March 25, 2013; revised 0ct. 17, 2013; accepted 0ct. 19, 2013.

Author contributions: B.S.F., A.V.K., and A.C.K. designed research; B.S.F., A.V.K., and N.H. performed research; B.S.F., A.V.K., and J.D.B. analyzed data; B.S.F., A.V.K., J.D.B., and A.C.K. wrote the paper.

Funding was provided by the National Institutes of Health, the Pew Scholars Program, and the McKnight Foundation. We thank Liza Shoenfeld for assistance with histology, Mattias Karlsson for assistance with image analysis, and members of the Kreitzer lab for helpful discussions. Some images were collected at the Nikon Imaging Center at University of California, San Francisco.

The authors declare not competing financial interests.

Correspondence should be addressed to Anatol C. Kreitzer, Gladstone Institute of Neurological Disease, 1650 Owens Street, San Francisco, CA 94158. E-mail: akreitzer@gladstone.ucsf.edu.

DOI:10.1523/JNEUROSCI.1278-13.2013

Copyright $\odot 2013$ the authors $\quad 0270-6474 / 13 / 3318531-09 \$ 15.00 / 0$
}

While the primate globus pallidus internal segment (GPi) is thought to control basal ganglia processing related to body and limb movements (Mushiake and Strick, 1995; Wenger et al., 1999), the rodent GPi (entopeduncular nucleus) is relatively far smaller (Smith et al., 1998), and the rodent SNr likely subserves many of the functions of the primate GPi. A recent study observed very similar pauses in $\mathrm{SNr}$ activity just before initiation of lateral head movements; these pauses were observed in a sector of $\mathrm{SNr}$ that projects to SC, and did not occur when movements were canceled (Schmidt et al., 2013). Similarly, other groups have identified SNr cells with decreased firing at points of movement initiation, although cells with excitatory responses were also common (Gulley et al., 1999, 2002; Meyer-Luehmann et al., 2002; Jin and Costa, 2010; Bryden et al., 2011; Fan et al., 2012).

Using cell type-specific targeting of channelrhodopsin-2 (ChR2), we developed a system in which we could optically activate either direct or indirect pathway striatal medium spiny neurons (dMSNs or iMSNs, respectively), while recording $\mathrm{SNr}$ activity and monitoring behavior in the open field. We previously showed that direct pathway activation for tens of seconds promotes locomotion, while indirect pathway activation suppresses movement (Kravitz et al., 2010). Using briefer pathway activations we sought to identify features of SNr activity, particularly changes in firing rate, which are essential for driving changes in locomotor behavior on a single trial basis. To best approximate physiological patterns of striatal activity, we used a constant illumination paradigm to avoid imposing patterned firing on stimulated neurons. We found that stimulating dMSNs and iMSNs produced diverse SNr firing rate responses, yet only the inhibited SNr neurons predicted locomotor activation during direct pathway activation. Conversely, excited neurons predicted 
motor suppression immediately after the onset of indirect pathway activation, while inhibited neurons were able to predict motor suppression only after more prolonged activation.

\section{Materials and Methods}

Subjects. BAC transgenic mouse lines that express Cre recombinase under control of the dopamine D1 receptor (D1-Cre EY217) and adenosine A2A (A2A-Cre KG139) receptor regulatory elements were obtained from GENSAT. Hemizygous BAC transgenic mice were crossed against wildtype C57BL/6 mice to generate hemizygotes used for experiments. Mice of either sex (D1-Cre: $n=2$ for striatal recordings, $n=5$ for SNr recordings, $n=7$ for Fos experiments, A2A-Cre: $n=2$ for striatal recordings, $n=4$ for SNr recordings, $n=13$ for Fos experiments) were used for recordings at $6-10$ weeks of age, and $4-18$ weeks of age for Fos experiments. All procedures were approved by the University of California, San Francisco Institutional Animal Care and Use Committee.

Viral expression of DIO-ChR2-YFP. We used commercially available AAV2/1-packaged DIO-ChR2-YFP or DIO-YFP to express ChR2-YFP or YFP alone in Cre-expressing cells (Sohal et al., 2009). We obtained virus from the viral vector cores at the University of North Carolina and the University of Pennsylvania. The final viral concentration was $4 \times 10^{12}$ to $3 \times 10^{13}$ viral particles per milliliter.

Stereotaxic surgery. For all procedures, anesthesia was induced with a mixture of ketamine and xylazine (100 mg ketamine plus $5 \mathrm{mg}$ xylazine per kilogram of body weight, i.p.) and maintained with isoflurane through a nose cone mounted on a stereotaxic apparatus (Kopf Instruments). The scalp was opened and holes were drilled in the skull bilaterally ( $+0.8 \mathrm{~mm}$ anteroposterior $(\mathrm{AP}), 1.5 \mathrm{~mm}$ mediolateral $(\mathrm{ML})$ from bregma). DIO-ChR2-YFP or DIO-YFP virus $(1 \mu \mathrm{l})$ was injected bilaterally into dorsomedial striatum $(-2.8 \mathrm{~mm}$ dorsoventral (DV) from top of brain) through a 33 gauge steel injector cannula (Plastics One) using a syringe pump (World Precision Instruments) that infused the virus over $5 \mathrm{~min}$. The injection cannula was left in place for $10 \mathrm{~min}$ following the injection and then slowly removed. For $\mathrm{SNr}$ recording experiments, bilateral ferrule-housed $105 \mu \mathrm{m}$ optical fibers were then implanted such that the fiber tips were located at the same coordinates as the viral injections. Additionally, an $\mathrm{SNr}$ craniotomy was drilled at the coordinates $-3.4 \mathrm{~mm}$ AP, $1.1 \mathrm{~mm} \mathrm{ML}$ from bregma, and a 16-channel microwire array $(35 \mu \mathrm{m}$ diameter, $7 \mathrm{~mm}$ long tungsten wires, $2 \times 8$ arrangement, $150 \mu \mathrm{m}$ spacing between wires, $200 \mu \mathrm{m}$ spacing between rows; Innovative Neurophysiology) was lowered into the SNr $4.5 \mathrm{~mm}$ DV from the brain surface. Dental adhesive (C\&B Metabond; Parkell) was used to fix the fiber optic ferrules in place and coat the surface of the skull. The ferrules and recording array were then cemented in place with dental acrylic (Ortho-Jet; Lang Dental). After the cement dried, the scalp was sutured shut. Surgeries for striatal recordings were performed similarly, except that after viral injection a fiber-coupled 32-channel microwire array (optrode; $4 \times 8$ arrangement; Innovative Neurophysiology) was unilaterally implanted in the striatum at the same coordinates used for injection. All surgical procedures were performed under aseptic conditions. For postoperation analgesia the mice received $2 \mathrm{mg} / \mathrm{kg}$ ketoprofen and $0.05 \mathrm{mg} / \mathrm{kg}$ buprenorphine. To allow time for viral expression, animals were housed for two to three weeks following surgery before recording or behavioral data were collected.

Histology. Following recording experiments, an electrolytic lesion was made at the electrode sites by individually passing $25 \mu \mathrm{A}$ of current through each microwire for $10 \mathrm{~s}$. The location of this lesion was verified to be in the striatum or $\mathrm{SNr}$ through post hoc histological analysis. Animals were killed with a lethal dose of ketamine and xylazine (400 mg ketamine plus $20 \mathrm{mg}$ xylazine per kilogram of body weight, i.p.) and transcardially perfused with first PBS and then $4 \%$ paraformaldehyde (PFA). Following perfusion, brains were left in 4\% PFA for 16-24 h and then moved to a $30 \%$ sucrose solution in PBS for 2-3 d. Brains were then frozen and cut into $30 \mu \mathrm{m}$ sections (either coronal or sagittal) with a sliding microtome (Leica Microsystems, model SM2000R) equipped with a freezing stage (Physitemp).

For Fos experiments, slices were preserved in cryoprotectant and stored at $-20^{\circ} \mathrm{C}$. The slices were stained using a standard diaminoben- zidine (DAB) protocol with $0.5 \%$ PBST for all washes, 15 min peroxide block, and $1 \mathrm{~h}$ blocking with normal goat serum and milk. The primary antibody (rabbit anti-c-fos; Millipore PC38) was used at a 1:1000 dilution and incubated on a shaker plate overnight at $4^{\circ} \mathrm{C}$. The secondary antibody (biotinylated goat anti-rabbit; Vector BA-1000) was incubated at a 1:1000 dilution for $2 \mathrm{~h}$ on a shaker plate at room temperature. A Vectastain ABC Kit was used (peroxidase standard PK-4000) as well as Vector's DAB peroxidase substrate kit (SK-4100) for development with DAB. The slices were mounted and stained with Luxol fast blue ( $1 \mathrm{~h}$ at $37^{\circ} \mathrm{C}$ ) as a counterstain to make nuclei recognition easier. Slides were differentiated with $0.05 \%$ lithium carbonate and alcohol, rinsed with $\mathrm{diH}_{2} \mathrm{O}$, and coverslipped with Cytoseal 60 .

Injection sites were verified by imaging unstained striatal slices from each animal for YFP. If the viral injection was not in the dorsomedial striatum the animal was excluded from the study. Slides were imaged using an Aperio ScanScope XT slide scanner at $20 \times$ magnification in bright field. Tissue was analyzed with the Aperio Spectrum software, wherein a region of interest was defined by the user and nuclei were counted within the region by the software.

Analysis of behavioral data. After laser-coupled optical fibers were coupled to the chronically implanted optical fibers, each mouse was placed in a square activity chamber $(41 \mathrm{~cm}$ side length) and video-monitored from above. The positions of the nose, tail, and center of mass of each mouse were tracked using ETHOVISION 7.1 software (Noldus). For recording experiments, animals were recorded for a $10 \mathrm{~min}$ baseline period before any laser illumination. Then the laser $(473 \mathrm{~nm}, 1.5 \mathrm{~mW}$ total power per fiber, $173 \mathrm{~mW} / \mathrm{mm}^{2}$ power at each fiber tip-measured with a PM100D optical power meter with an S120C sensor; Thorlabs) was activated in a series of 200 trials. Each of the first 100 trials consisted of $1 \mathrm{~s}$ laser ON periods followed by $9 \mathrm{~s}$ off. The second 100 trials consisted of $100 \mathrm{~ms}$ laser ON periods followed by $9 \mathrm{~s}$ off. Center-point velocity was binned at 50 $\mathrm{ms}$, and latency to a significant change in velocity during illumination was computed as the first ON bin outside the $99.5 \%$ confidence interval for the $1 \mathrm{~s}$ Pre period binned data. Locomotion was defined as periods when the velocity of the animal's center point averaged $>2 \mathrm{~cm} / \mathrm{s}$ for at least $0.5 \mathrm{~s}$. The beginning of such a period is defined as a "locomotor start." For direct pathway success/failure analysis, behavioral trials were separated into failures in which there was no locomotor start occurring $2 \mathrm{~s}$ after the beginning of illumination, and successes in which a locomotor start did occur. Immobility was defined as continuous periods of time during which the average pixel change of the entire video image was $<2 \%$ for at least $1 \mathrm{~s}$. This definition was very strict, such that any movement of the head, limbs, or tail would not be scored as immobility. For indirect pathway success/failure analysis, behavioral trials were separated into failures in which there was no immobility start occurring $2 \mathrm{~s}$ after the beginning of illumination, and successes in which an immobility start did occur. The center-point velocity of the animal and the initiation of ambulation and immobility periods were calculated using ETHOVISION 7.1. All behavioral data were collected concurrently with the neural recordings.

For Fos experiments, mice were subjected to $3 \mathrm{~d}$ of habituation followed by the experimental protocol. Habituation consisted of $60 \mathrm{~min}$ each day in a sham box in the behavior room plugged into a dummy cannula. The experimental day began with $2 \mathrm{~h}$ of habituation followed by the stimulation protocol: $60 \mathrm{~min}$ total of $30 \mathrm{~s}$ laser ON, $30 \mathrm{~s}$ laser OFF at $208 \mathrm{~mW} / \mathrm{mm}^{2}$. Rotational data were collected and analyzed using Noldus EthoVision XT. After the stimulation protocol the mice were returned to the home cage for $60 \mathrm{~min}$ before perfusion.

Analysis of neural recordings. Voltage signals from each microwire were bandpass filtered, such that activity between 150 and $8000 \mathrm{~Hz}$ was analyzed as spiking activity. Data were amplified, processed, and digitally captured using commercial hardware and software (Plexon). Single units were discriminated manually, with the aid of principal component analysis (OFFLINE SORTER 3.0.1; Plexon). SNr neurons were characterized by rapid extracellular action potential kinetics and large single-unit amplitudes (466 $\pm 47 \mu \mathrm{V}, n=60$ units). High-quality unit isolation was achieved, as assessed by measures of spike-sorting quality (Nicolelis et al., 2003; Davies-Bouldin index $0.30 \pm 0.02$, J3 statistic $3.5 \pm 1.1, n=10$ wires with multiple single units). All analysis of neural data was derived from $1 \mathrm{~s}$ laser illumination trials. For characterization of direct and indi- 
A

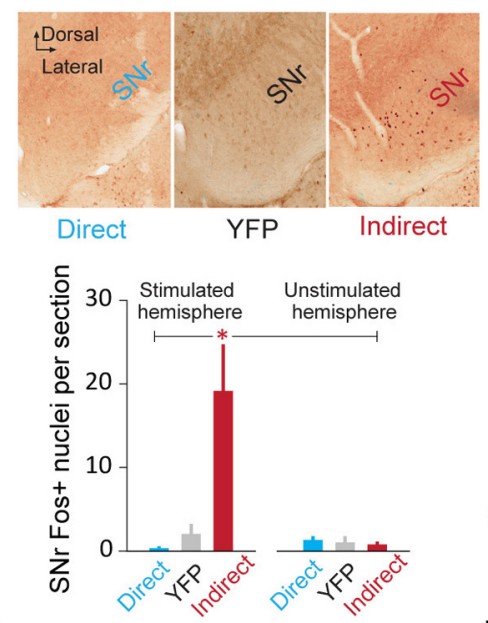

C
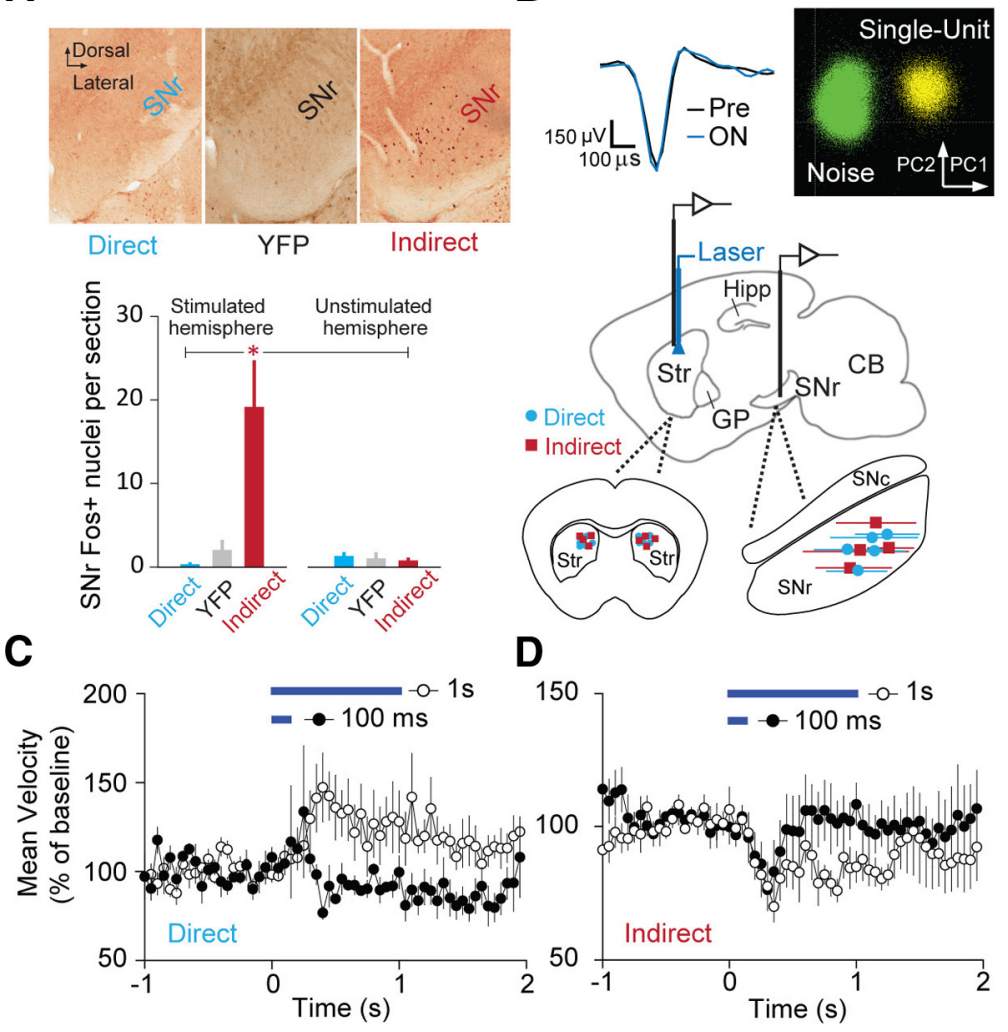

Figure 1. Optogenetic activation of basal ganglia circuits. A, Top, Coronal SNr sections stained for Fos following direct or indirect pathway stimulation. A coronal section for a YFP control experiment is shown for comparison. Bottom, Quantification of Fos ${ }^{+}$ nuclei for stimulated and unstimulated direct pathway ( $n=4$ hemispheres stim, $n=3$ unstim), YFP ( $n=5$ hemispheres stim, $n=3$ unstim), and indirect pathway ( $n=7$ hemispheres stim, $n=8$ unstim) groups. $\boldsymbol{B}$, Top left, Average extracellular action potential waveforms for a representative SNr neuron in the Pre and ON periods. Top right, $2 D$ PCA clustering of spike waveforms for the same $\mathrm{SNr}$ unit. Middle, Sagittal schematic (1.7 mm lateral from midline) showing placement of bilateral optical fibers in dorsomedial striatum with unilateral recording array either in striatum or SNr. Bottom left, Coronal striatal schematic showing bilateral placement of optical fibers for direct $(n=5)$ and indirect $(n=4)$ pathway experiments. Bottom right, Sagittal SNr schematic showing the extent of recording sites for direct $(n=5)$ and indirect $(n=4)$ pathway experiments. $C$, Time courses of mouse open-field velocity in response to $100 \mathrm{~ms}$ or $1 \mathrm{~s}$ direct pathway activation (denoted by blue bars; $n=5$ mice). $\boldsymbol{D}$, Mean velocity for $100 \mathrm{~ms}$ or $1 \mathrm{~s}$ indirect pathway activation ( $n=4$ mice). CB, Cerebellum; SNc, substantia nigra pars compacta. first computed the percentage change in firing rate from Pre to $\mathrm{ON}$ for each unit. The sets of firing rate changes for all mice in a given pathway-activation experiment were then analyzed for statistically significant differences using the Kruskal-Wallis test.

Group mean firing rate curves for success and failure trials (defined above) were calculated using all single-unit data in either $10 \mathrm{~ms}$ (indirect pathway) or $50 \mathrm{~ms}$ (direct pathway) bins. Receiver operating characteristic (ROC) curves were computed for excited and inhibited units across all behavioral trials $(n=100$ trials per unit) using average firing rates within $100 \mathrm{~ms}$ or $1 \mathrm{~s}$ windows as indicated. For both direct and indirect pathway experiments, the positive state was defined as behavioral success (presence of locomotor or immobility start) and the negative state was defined as behavioral failure (absence of locomotor or immobility start). Single units were considered to be predictive if average firing rate changes exhibited significant discriminability between behavioral trial types (area under the ROC curve $>0.5$ with $p<0.05$ ). Average response ROC curves for the inhibited and excited subpopulations were calculated by computing the subpopulation firing rate $(\mathrm{FR})$ average $=(1 /$ number of subpop single units) ${ }^{*} \Sigma \mathrm{FR}_{\text {subpop single unit }}$ for each mouse across all behavioral trials $(n=500$ total trials for direct pathway and $n=400$ total trials for indirect pathway). All ROC curves are displayed such that they lie above the line of no-discriminability; the direction of predictive firing rate changes is noted in the text. All analysis was performed using Offline Sorter v3 (Plexon), NeuroExplorer 4.091 (Nex Technologies), Excel 2010 (Microsoft), MATLAB (MathWorks), and SigmaPlot 11.2 (Systat Software).

\section{Results}

\section{Striatal stimulation modulates $\mathrm{SNr}$} Fos expression

We first obtained histological evidence that rect pathway striatal responses, we first identified putative dMSNs and iMSNs as those units having a significant change in firing rate within 40 $\mathrm{ms}$ of the beginning of the laser pulse (at $1 \mathrm{~mW}$ total power per fiber, 116 $\mathrm{mW} / \mathrm{mm}^{2}$ ). Latency to a significant change in MSN firing rate elicited by illumination was calculated by binning the firing rate of an individual unit at $5 \mathrm{~ms}$ over the interval -1 to $+1 \mathrm{~s}$. The time of the first $\mathrm{ON}$ period bin outside the $99.7 \%$ confidence interval of the average binned $1 \mathrm{~s}$ Pre period firing rate was considered to be the response latency. Group mean responses were generated by averaging the responses of the identified dMSNs and iMSNs. For characterization of SNr responses to direct and indirect pathway stimulation, we computed group mean firing rate curves by simply averaging all single-unit firing rates (in hertz). Latency to a significant change in $\mathrm{SNr}$ firing rate elicited by illumination (at 1.5 $\mathrm{mW}$ total power per fiber, $173 \mathrm{~mW} / \mathrm{mm}^{2}$ ) was calculated by binning the firing rate of an individual unit at $20 \mathrm{~ms}$ over the interval -1 to $+1 \mathrm{~s}$. The time of the first ON period bin outside the $99.5 \%$ confidence interval computed from the binned $1 \mathrm{~s}$ Pre period firing rate was considered to be the response latency. Pre period mean firing rates were calculated as the average firing rate over the entire $1 \mathrm{~s}$ period immediately preceding illumination. ON period mean firing rates were calculated as the average firing rate of the entire $1 \mathrm{~s}$ period during illumination. Comparisons of the magnitudes of illumination-induced changes in firing rates between subpopulations of inhibited and excited cells were performed by the Wilcoxon rank sum test. To determine whether firing rate changes were similar across mice for either direct or indirect pathway activation, we optical stimulation of ChR2-expressing direct pathway (dopamine D1 receptor expressing) dMSNs or indirect pathway (adenosine A2A receptor expressing) iMSNs modulates activity patterns in the $\mathrm{SNr}$. To robustly activate immediate early gene expression, we subjected mice to a $60 \mathrm{~min}$ session of alternating $30 \mathrm{~s}$ periods of laser $\mathrm{OFF}$ and laser $\mathrm{ON}$, and assayed the number of c-Fos-positive nuclei as an indicator of recent SNr activity (Sagar et al., 1988; Sheng et al., 1990; Yizhar et al., 2011). We found that indirect pathway activation significantly potentiated Fos expression, consistent with the proposed excitatory nature of this circuit. Direct pathway activation did not significantly reduce expression (Fig. $1 A$ ), likely due to the low baseline expression of c-Fos in the $\mathrm{SNr}$ (mean number of $\mathrm{SNr} \mathrm{Fos}^{+}$ nuclei per section: stimulated-direct $0.33, n=4$ mice, YFP 2.03, $n=$ 5, indirect 19.1, $n=7$; unstimulated-direct 1.33, $n=3$, YFP 1.04, $n=8$, indirect $0.79, n=8$, ANOVA $F=6.7, p<0.01$, direct vs indirect $p<0.05$, direct vs YFP $p=0.98$, indirect vs YFP $p<0.05$, Tukey's post hoc test).

\section{Activation of direct and indirect pathways oppositely regulate locomotion}

To identify the behaviorally relevant effects of direct and indirect pathway stimulation on basal ganglia output, we recorded striatal and $\mathrm{SNr}$ single-unit activity in mice during bilateral optical acti- 
vation of dMSNs or iMSNs (Fig. 1B). This system enabled us to obtain high-quality neural recordings in both baseline (Pre) and laser ON conditions, while simultaneously monitoring the locomotor effects of pathway activation as mice behaved ad libitum in the open field.

We found that bilateral direct pathway activation for $1 \mathrm{~s}$ was sufficient to significantly increase the average center-point velocity of the animal (Fig. 1C), with a mean latency of $300 \mathrm{~ms}(n=5$ mice). Illumination (100 ms) did not reliably evoke a velocity change ( $n=5$ mice). Consistent with our previous results, $1 \mathrm{~s}$ indirect pathway illumination induced a significant decrease in velocity (Fig. $1 D)$, with a latency of $350 \mathrm{~ms}(n=4$ mice). Interestingly, indirect pathway activation for $100 \mathrm{~ms}$ also provoked a decrease in velocity with a similar onset time course to that for $1 \mathrm{~s}$ illumination (latency $=300 \mathrm{~ms}, n=4$ mice). These results suggest that brief, selective manipulations of basal ganglia output can drive strong behavioral effects. For these reasons, we chose to analyze single-unit data on these short timescales, with the goal of determining how direct and indirect pathway modulation of $\mathrm{SNr}$ activity regulates locomotion.

\section{Direct pathway activation selectively inhibits subsets of SNr neurons}

To investigate how the direct pathway modulates $\mathrm{SNr}$ single-unit activity, we first verified that our stimulation conditions were sufficient to excite striatal dMSNs in behaving D1-Cre mice. Indeed, we found that low-power striatal illumination at $1 \mathrm{~mW}$ total power per fiber $\left(116 \mathrm{~mW} / \mathrm{mm}^{2}\right)$ elicited time-locked increases in firing rate in a subset of recorded striatal cells (Fig. $2 \mathrm{~A}$, left), which we identified as putative dMSNs ( $n=6$ neurons). The average $\mathrm{SNr}$ firing rate response displays similar kinetics (Fig. $2 A$, right; $n=27 \mathrm{SNr}$ neurons). Interestingly, we found that direct pathway activation alone was sufficient to produce robust inhibition of a subset of SNr neurons ( $n=11$ of 27 neurons), along with excitation of a different subset of cells ( $n=15$ of 27 neurons). Figure 2, $B$ and $C$, shows example responses and group averages for these cell subpopulations, demonstrating robust firing rate modulation both within and across trials. Thus in total, 26 of 27 recorded SNr neurons had a significant change in firing rate in response to laser stimulation, suggesting that our stimulation conditions strongly modulated $\mathrm{SNr}$ output. Firing rate changes were not statistically different across animals, demonstrating that the responses we observed were consistent $(H=8.2$, $n=27$ cells across 5 mice, $p=0.083$, Kruskal-Wallis test). Median illumination-induced changes in firing rate did not differ in magnitude between excited and inhibited cells (Fig. 2D; inhibited Pre median FR 20.7 Hz, ON median FR 6.0 Hz, $n=11$ cells; excited Pre median FR 10.2 Hz, ON median FR $12.7 \mathrm{~Hz}, n=15$ cells, $p>0.05$, rank sum test).

Excited neurons had significantly lower Pre period mean firing rates than inhibited neurons (Fig. $2 D ; p<0.01$, rank sum test). They also exhibited a trend toward longer response latencies, suggesting that while direct pathway-mediated inhibition is a monosynaptic effect (Ryan et al., 1986), excitation may be more likely disynaptic or polysynaptic (inhibited median latency $=20$ $\mathrm{ms}, n=11$ neurons, excited median latency $=60 \mathrm{~ms}, n=15$ neurons, $p=0.069$, rank sum test).

\section{Indirect pathway activation selectively excites subsets of SNr neurons}

In contrast to the direct pathway, the indirect pathway has been hypothesized to serve as a brake on motor behavior via STNmediated excitation of basal ganglia output (Li et al., 2008; Eagle
A
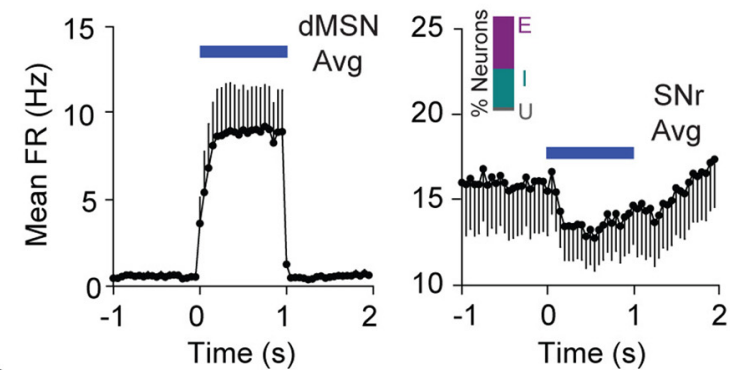

B
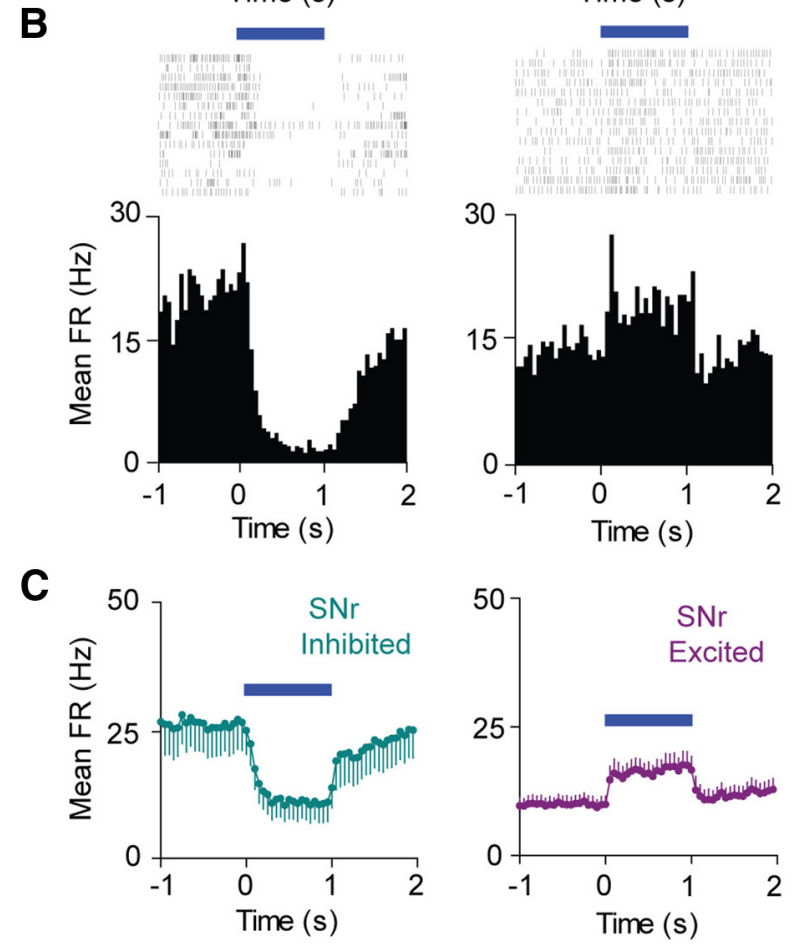

D
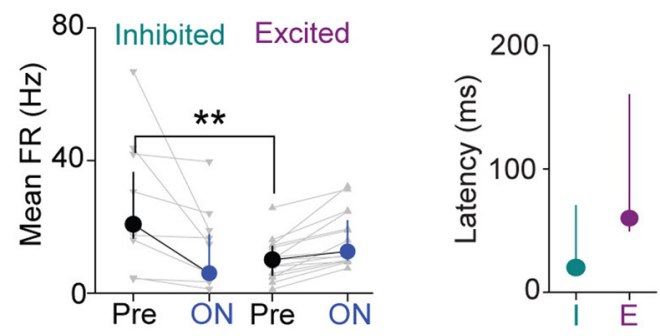

Figure 2. Direct pathway activation selectively inhibits subsets of SNr neurons. $\boldsymbol{A}$, Left, Average time course ( \pm SEM) of striatal dMSN activation in response to 1 s illumination $(n=6$ neurons). Right, Average time course ( \pm SEM) of SNr neuron modulation in response to $1 \mathrm{~s}$ striatal illumination ( $n=27$ neurons). Time is binned at $50 \mathrm{~ms}$ in each case. Inset, Bar graph showing percentage of neurons excited $(E)$, inhibited $(I)$, or without significant response $(U)$ to direct pathway activation. $\boldsymbol{B}$, Left, Raster plot and perievent histogram for an SNr neuron strongly inhibited by $1 \mathrm{~s}$ direct pathway activation (blue bar). Right, Raster plot and perievent histogram for an SNr neuron excited by $1 \mathrm{~s}$ direct pathway activation. $\boldsymbol{C}$, Left, Average time course for inhibited neurons ( $n=11$ neurons). Right, Average time course for excited neurons ( $n=15$ neurons). Error bars indicate SEM. Time is binned at $50 \mathrm{~ms}$. D, Left, Changes in mean FR from Pre to $0 \mathrm{~N}$ conditions for inhibited cells and excited cells. Individual cells are shown in gray, and the group medians ( $\pm \mathrm{IQR}$ ) are shown in black or blue. Right, Median ( $\pm \mathrm{IQR}$ ) latencies to a significant change in firing rate for both inhibited and excited cell populations ( $n=11$ inhibited, $n=15$ excited).

et al., 2011; Jahfari et al., 2011). To assess this hypothesis, we first confirmed that we could activate striatal iMSNs ( $n=6$ neurons; Fig. 3A). Consistent with the prediction that indirect pathway activation should enhance SNr firing, we observed a population 
A
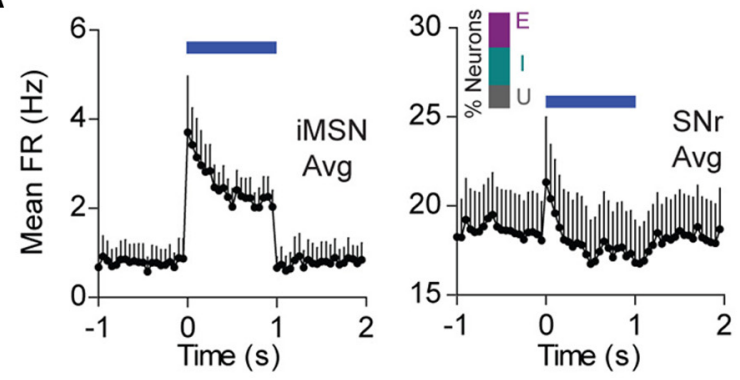

B
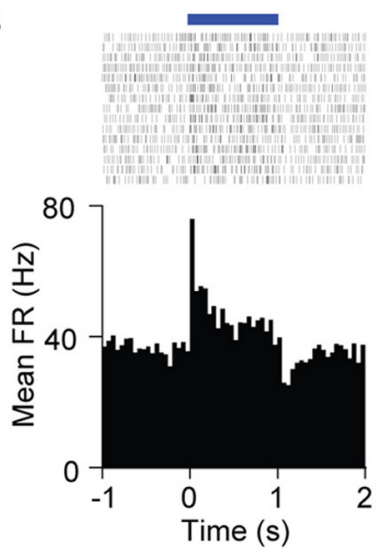

C

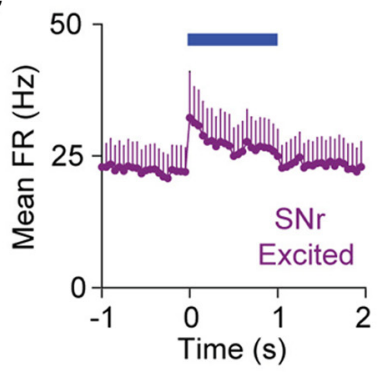

D

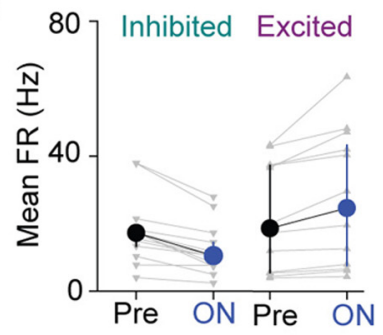

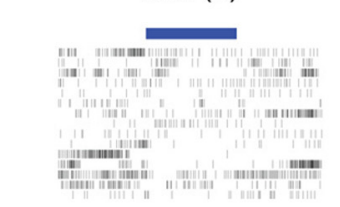

80
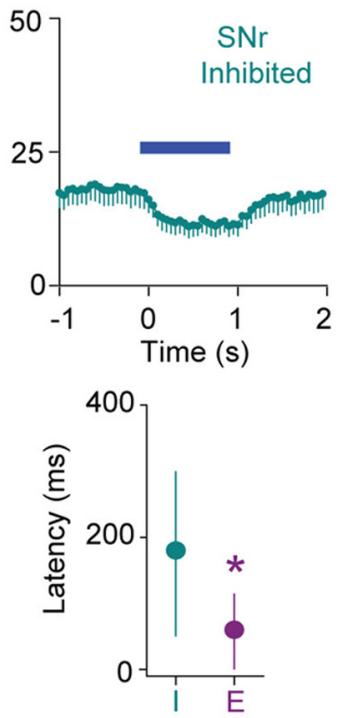

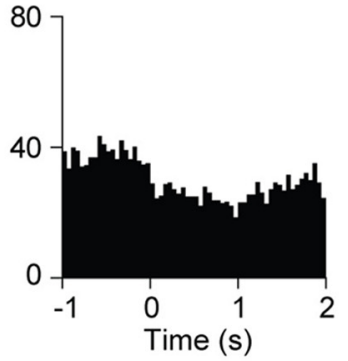

Figure 3. Indirect pathway activation selectively excites subsets of $\mathrm{SNr}$ neurons. A, Left, Average time course ( \pm SEM) of striatal iMSN activation in response to 1 s illumination ( $n=6$ neurons). Right, Average time course ( \pm SEM) of SNr neuron modulation in response to $1 \mathrm{~s}$ striatal illumination. Inset, Bar graph showing percentage of neurons excited (E), inhibited (I), or without significant response (U) to indirect pathway activation. $\boldsymbol{B}$, Left, Raster plot and perievent histogram for an $\mathrm{SNr}$ neuron strongly excited by $1 \mathrm{~s}$ indirect pathway activation. Right, Raster plot and perievent histogram for an $\mathrm{SNr}$ neuron inhibited by 1 s indirect pathway activation. C, Left, Average time course for excited neurons ( $n=12$ neurons). Right, Average time course for inhibited neurons ( $n=13$ neurons). Error bars indicate SEM. Time is binned at $50 \mathrm{~ms}$. D, Left, Changes in mean FR from Pre to $\mathrm{ON}$ conditions for inhibited cells and excited cells. Individual cells are shown in gray, and the group medians ( $\pm I Q R)$ are shown in black or blue. Right, Median ( $\pm I Q R)$ latencies to a significant change in FR for both inhibited and excited cell populations ( $n=12$ excited, $n=13$ inhibited).

of $\mathrm{SNr}$ cells that are strongly and rapidly excited by $1 \mathrm{~s}$ indirect pathway activation ( $n=12$ neurons). SNr excitation and iMSN activation both exhibit depressing time courses, suggesting that the indirect pathway circuit can relay temporal changes in iMSN

firing rates (Fig. $3 A, B$ ). In addition to cells with excitatory responses, we also observed a class of neurons that are inhibited by indirect pathway activation (Fig. $3 C$, right; $n=13$ inhibited neurons). Median illumination-induced firing rate changes did not differ in magnitude between the excited and inhibited cells (Fig. 3D; excited Pre median FR 18.7 Hz, ON median FR 24.7 Hz, $n=$ 12 cells; inhibited Pre median FR $17.3 \mathrm{~Hz}$, ON median FR 10.5 $\mathrm{Hz}, n=13$ cells, $p>0.05$, rank sum test). Similar to our observations for direct pathway activation, firing rate changes were not statistically different across animals $(H=3.4, n=33$ cells across 4 mice, $p=0.33$, Kruskal-Wallis test).

Excited cells had significantly shorter response latencies than inhibited cells (Fig. 3D; excited median latency $=60 \mathrm{~ms}, n=12$ cells; inhibited median latency $=180 \mathrm{~ms}, n=13$ cells; $p<0.05$, rank sum test). In contrast to our results for direct pathway activation, excited and inhibited cells did not have significantly different Pre period firing rates (Fig. $3 D ; p=0.77$, rank sum test).

\section{Inhibited SNr cells predict movement initiation}

Direct pathway activation for $1 \mathrm{~s}$, but not $100 \mathrm{~ms}$, elicits, on average, an increase in movement (Fig. 1C). Frequently, these increases in movement occur as a locomotor start, defined as the beginning of a period in which the center point of the mouse moved at a rate of $2 \mathrm{~cm} / \mathrm{s}$ for at least $0.5 \mathrm{~s}$ (Fig. $4 A$ ). However, the ability of a particular stimulation trial to elicit a locomotor start is variable. We exploited this variability to identify features of $\mathrm{SNr}$ activity that could distinguish between trials that elicited a locomotor start (success trials), and those that did not (failure trials; Fig. $4 B, C$ ). Because direct pathway stimulation elicits a variety of neural effects, we hypothesized that this approach could allow us to separate the aspects of $\mathrm{SNr}$ activity with a strong relationship to locomotor starts from those that may be important for other processes. By analyzing differences in single-unit activity between trial types, we identified cells that had differing responses to direct pathway activation for failures and successes. Figure $4 D$ shows perievent rasters and histograms for an example SNr neuron that exhibited a lower firing rate for success trials.

We sought to determine whether SNr cells were able to discriminate between success and failure trials on a single trial basis. To answer this question, we calculated ROC curves (Quiroga et al., 2005) for single units across all behavioral trials. We found that during illumination, $8 / 11$ inhibited neurons and 5/15 excited neurons had areas under the ROC curve (AUC) significantly $>0.5$, demonstrating that single neurons were indeed able to distinguish between success and failure trials. In the Pre period, 4/11 inhibited cells and 2/15 excited cells demonstrated selectivity for behavioral outcome (Fig. 4G). We also computed ROC curves for the averaged inhibited and excited subpopulation responses. In the Pre period, the inhibited subpopulation showed robust discriminating ability (AUC $0.65, p<0.001, n=11$ units), while the excited subpopulation showed lesser discriminating ability (AUC 0.57, $p<0.01, n=15$ units; Fig. $4 E, H)$. In the ON period, the inhibited subpopulation showed discriminating ability (AUC 0.62, $p<0.001, n=11$ units), while the excited subpopulation $\operatorname{did} \operatorname{not}(\mathrm{AUC} 0.53, p>0.05, n=15$ units; Fig. $4 F, H$ ). In all cases, significantly discriminating subpopulations fired less for success trials, consistent with a role for inhibition in driving locomotor starts. These results demonstrate that the inhibited cell subpopulation strongly predicts locomotor initiation, while the excited subpopulation is less selective. 


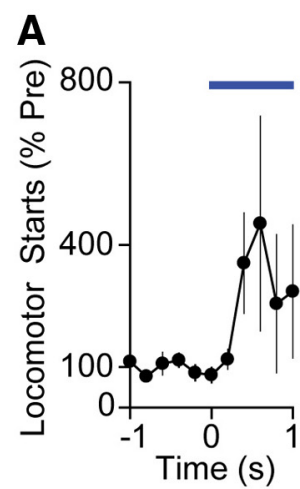

E

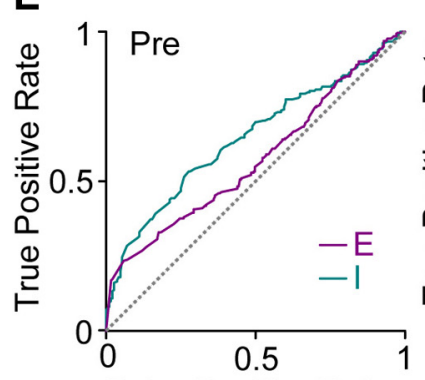

False Positive Rate

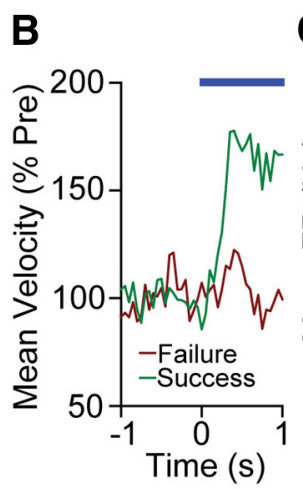

$\mathbf{F}$
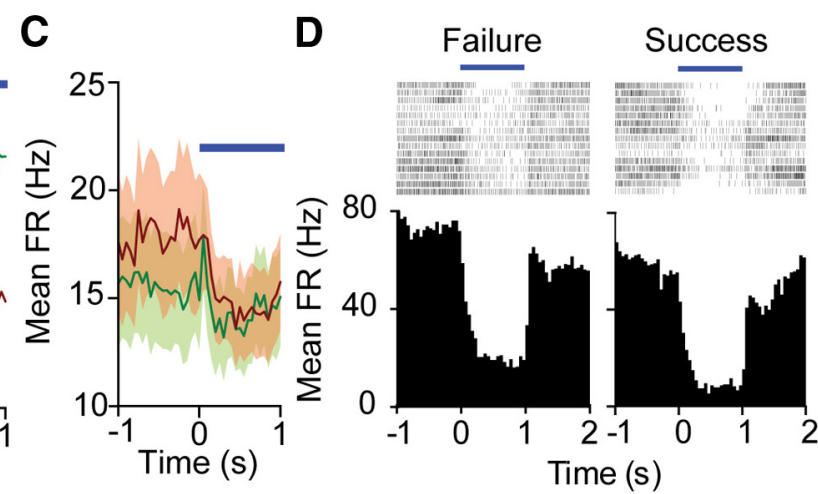

$\mathbf{H}$

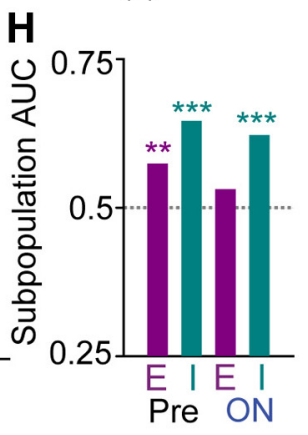

Figure 4. Inhibited SNr cells predict movement initiation. $\boldsymbol{A}$, Mean ( \pm SEM) locomotor starts per animal $(n=5)$ for $1 \mathrm{~s}$ dMSN activation. $\boldsymbol{B}, \mathrm{Group}$ mean normalized velocity for trials that do not elicit a locomotor start ("failures"; red) and trials that do elicit a locomotor start ("successes"; green), indicating the abrupt increase in velocity for success trials. C, Population mean FR for failure and success trials ( $n=27$ neurons). SEM is shaded in corresponding colors. $\boldsymbol{D}$, Raster plots and perievent histograms for the same SNr neuron in failure trials (left) and success trials (right). $\boldsymbol{E}$, Left, ROC curves for excited ( $E ; n=15$ neurons) and inhibited (I; $n=11$ neurons) subpopulation responses in the Pre period. The gray dashed line is defined by the equation true positive rate $=$ false positive rate and represents lack of discrimination between behavioral states. $\boldsymbol{F}, \mathrm{ROC}$ curves for excited (E; $n=15)$ and inhibited (I; $n=11)$ subpopulations in the $0 \mathrm{~N}$ period. $\boldsymbol{G}$, Percentage of individual neurons with significant discriminability for behavioral outcome in the Pre and ON periods (E, excited, $n=15$ neurons; I, inhibited, $n=11$ neurons). $\boldsymbol{H}$, Area under the ROC curve (AUC) for excited $(\mathrm{E} ; n=15$ neurons) and inhibited ( $\mathrm{l} ; n=11$ neurons) subpopulation responses in the Pre and ON periods. The gray dashed line represents $\mathrm{AUC}=0.5$.

\section{Excited SNr neurons predict movement suppression}

We performed a similar success/failure analysis on our indirect pathway dataset. Because indirect pathway activation suppresses movement, we defined success trials as those in which the mouse became immobile and failure trials as those in which it did not (Fig. 5A-C). The average indirect pathway response (Fig. $3 A$ ) is rapid, and $100 \mathrm{~ms}$ indirect pathway activation produces a robust decrease in movement (Fig. 1D), so we focused our analysis on $100 \mathrm{~ms}$ intervals at the start of (and immediately before) $1 \mathrm{~s}$ indirect pathway activation trials. As with direct pathway activation, we were able to identify $\mathrm{SNr}$ cells that had activity patterns that differed between trial types. Figure $5 D$ shows perievent rasters and histograms for an example $\mathrm{SNr}$ neuron that is more strongly excited for success trials.

In contrast to our direct pathway results, in which the majority of inhibited units showed single trial behavioral selectivity by ROC analysis, none (0/13) of the inhibited units showed selectivity in the ON period, and only $2 / 13$ showed selectivity in the Pre period. Similarly, of 12 excited units, only 1 showed selectivity during the $\mathrm{ON}$ period and none showed selectivity in the Pre period. (Fig. $5 G$ ). Despite lack of selectivity on the single neuron level, the Pre period excited cell average response showed strong discriminability (AUC $0.62, p<0.001, n=12$ units). The inhibited cell average response did not (inhibited AUC 0.52, $p>0.05$, $n=13$ units; Fig. 5E,H). Similarly, during illumination, the excited subpopulation showed selectivity (AUC 0.60, $p<0.01$, $n=12$ units) while the inhibited subpopulation did not (AUC 0.53, $p>0.05, n=13$ units; Fig. $5 F, H$ ). In both $\mathrm{ON}$ and Pre conditions, the excited cell subpopulation fired more for success trials, suggesting that excitation drives motor suppression.
We additionally analyzed our indirect pathway data over the entire $1 \mathrm{~s}$ illumination period. We hypothesized that broadening the window may increase the discriminating ability of individual neurons. However, we found that during this $1 \mathrm{~s}$ ON period, only 2/12 excited units and 2/13 inhibited units demonstrated behavioral selectivity, consistent with poor behavioral discrimination on the single neuron level. The excited cell subpopulation response retained its ability to discriminate during this $1 \mathrm{~s}$ window (AUC 0.57, $p<0.05, n=12$ units). Interestingly, the inhibited cell subpopulation also demonstrated behavioral selectivity during this window (AUC 0.63, $p<0.001$ ), consistent with the slower kinetics of inhibitory responses during indirect pathway activation (data not shown). While the excited subpopulation fired more for success trials, the inhibited subpopulation fired less for success trials during the $1 \mathrm{~s}$ window.

\section{Discussion}

In this study, we used recent advances in optogenetic technology to investigate the contributions of direct and indirect pathways to basal ganglia circuit function. We selectively targeted the pathways originating in the dorsomedial striatum, which have been shown to have a greater influence on locomotion than those in the dorsolateral striatum (Durieux et al., 2012). Consistent with our previous experiments using longer activation times, brief direct pathway activation elicited locomotor activation, while indirect pathway activation strongly suppressed movement.

We sought to evoke naturalistic firing patterns in MSNs by delivering constant low-intensity illumination. Rather than imposing structured firing upon MSNs, we hypothesize that this approach enables subthreshold depolarization of MSNs. This is 
A

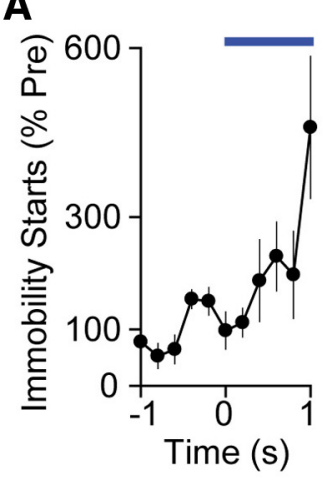

E

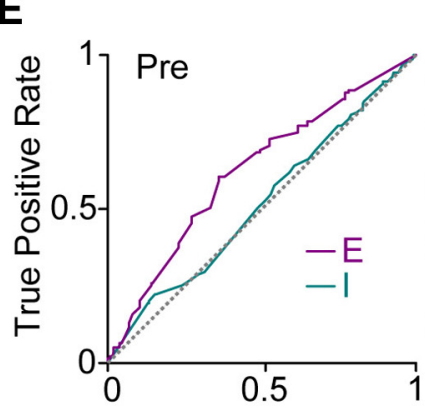

False Positive Rate
B C

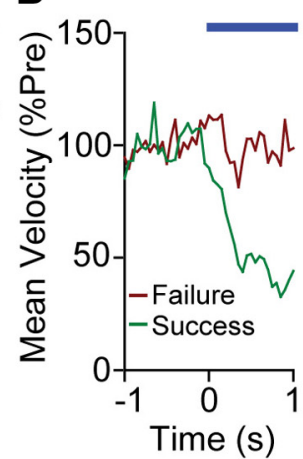

F

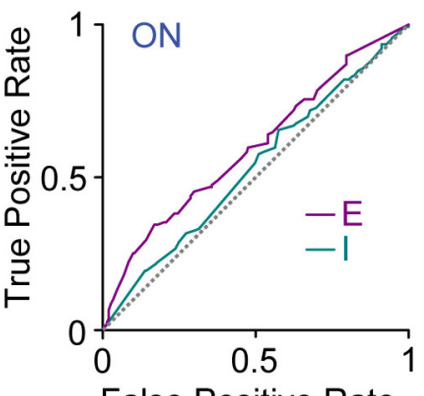

False Positive Rate

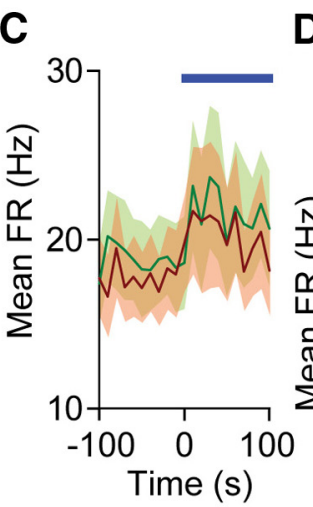

D Failure

Failure Success
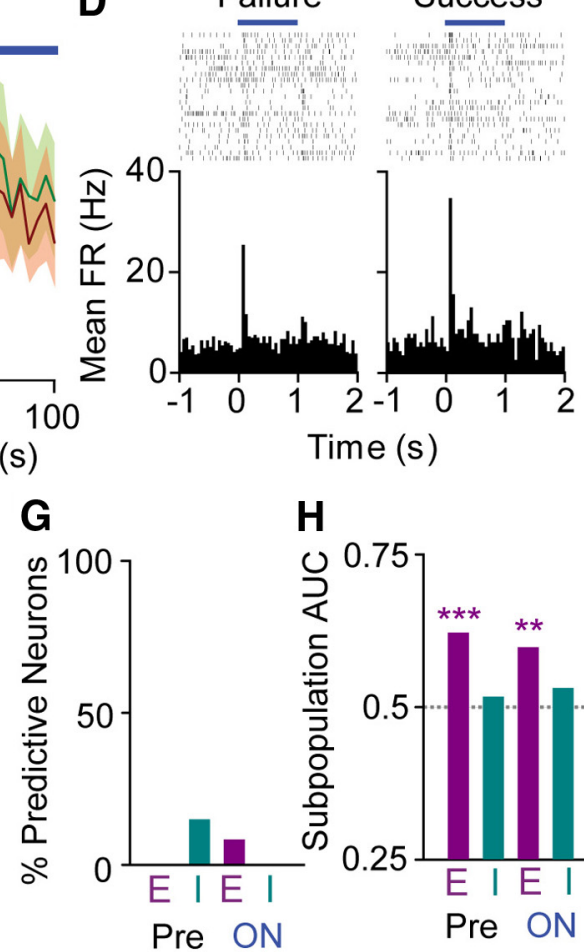

H

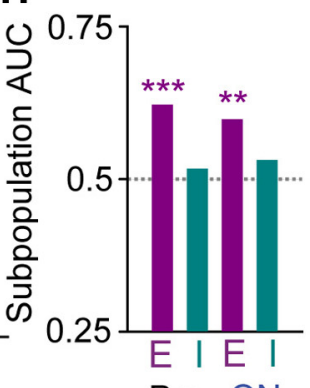

Figure 5. Excited SNr neurons predict motor suppression. $A$, Mean ( \pm SEM) immobility starts per animal $(n=4)$ for 1 siMSN activation. $B$, Group mean normalized velocity for all trials that do not elicit an immobility start ("failures"; red) and those that do elicit an immobility start ("successes"; green). C, Population mean FR for failure and success trials ( $n=33$ neurons). SEM shaded in corresponding colors. $\boldsymbol{D}$, Raster plots and perievent histograms for the same SNr neuron in failure trials (left) and success trials (right). $\boldsymbol{E}$, ROC curves for excited (E; $n=12$ neurons) and inhibited (I; $n=13$ neurons) subpopulations in the Pre period. The gray line is defined by the equation true positive rate $=$ false positive rate and represents lack of discrimination between behavioral states. $\boldsymbol{F}$, ROC curves for excited (E; $n=12$ neurons) and inhibited ( $; ; n=13$ neurons) subpopulations in the ON period. $\mathbf{G}$, Percentage of individual neurons with significant discriminability for behavioral outcome in the Pre and ON periods (E, excited, $n=12$ neurons; I, inhibited, $n=13$ neurons). $\boldsymbol{H}$, Area under the ROC curve (AUC) for excited (E; $n=12$ neurons) and inhibited (I; $n=13$ neurons) subpopulation responses in the Pre and ON periods. The gray dashed line represents $A U C=0.5$.

predicted to modestly increase firing rate, while still allowing synaptic inputs to control spike timing. Indeed, we found that this illumination scheme produced firing rate changes in striatal projection neurons that are similar to those observed during spontaneous movement initiation (Isomura et al., 2013).

Direct pathway activation evoked inhibitory and excitatory effects in different $\mathrm{SNr}$ cell populations. One potential explanation for this result is that direct pathway activation relieves $\mathrm{SNr}$ lateral inhibition on excited cells by inhibiting laterally connected neighbors with strong striatal inputs. $\mathrm{SNr}$ neurons have been shown to form local synapses, providing evidence for this possibility (Deniau et al., 1982; Mailly et al., 2003). While SNr lateral inhibition is an attractive explanation for these results, it is possible that other circuit effects could be at play within the striatum, $\mathrm{SNr}$, or complex loops involving multiple basal ganglia nuclei. Regardless of the mechanism for direct pathway-mediated $\mathrm{SNr}$ excitation, it is intriguing that direct pathway activation alone is capable of reproducing previous findings that both inhibited and excited cells are prevalent at points of movement initiation. This has been shown in rodent SNr (Gulley et al., 1999, 2002; MeyerLuehmann et al., 2002; Bryden et al., 2011), as well as the primate GPi (Georgopoulos et al., 1983; Anderson and Horak, 1985; Mitchell et al., 1987; Brotchie et al., 1991; Mink and Thach, 1991), suggesting that this is a common pattern of activity in basal ganglia output across vertebrates.

Indirect pathway stimulation induced $\mathrm{SNr}$ single-unit activity distinct from that observed for direct pathway stimulation. On average, $1 \mathrm{~s}$ indirect pathway activation produced rapid, but de- pressing, excitation of a subset of neurons, followed by slower onset inhibition in a different population of cells. The median latency for inhibited cells was threefold greater than that for excited cells, suggesting that SNr excitation is a direct effect of STN excitation (or GP-mediated disinhibition), while inhibition is a slower effect that occurs due to lateral inhibition within the $\mathrm{SNr}$ or elsewhere within the basal ganglia.

Given the diverse effects of pathway activation on SNr activity, we sought to determine what particular elements of this activity were closely associated with behavioral effects. Using ROC analysis, we first demonstrated that during direct pathway activation the majority of inhibited single units, as well as the average inhibited cell response, showed selectivity for behavioral outcome. In contrast, the excited cell subpopulation showed lesser discriminability on both the single neuron and average subpopulation response level. Sato and Hikosaka (2002) obtained an analogous result in a study of primate saccade behavior, in which $\mathrm{SNr}$ cells that decreased their firing predicted saccade direction, but cells that increased their firing did not. Anatomically, the direct pathway projection from striatum to basal ganglia output is highly convergent (Hazrati and Parent, 1992; Parent and Hazrati, 1993), making this circuit well suited for inhibition of particular $\mathrm{SNr}$ target cells, and activation of specific motor programs in downstream regions.

Interestingly, while indirect pathway activation also elicited inhibitory and excitatory effects in $\mathrm{SNr}$, only the excited cell average response predicted motor suppression in the $100 \mathrm{~ms}$ immediately after, and before, the onset of pathway activation. In 
contrast to our results for direct pathway activation, in which the majority of individual inhibited neurons showed behavioral selectivity, individual $\mathrm{SNr}$ neurons were poor discriminators of behavioral outcome during indirect pathway activation. This is consistent with theoretical predictions that motor suppression should arise when excited $\mathrm{SNr}$ cells prevent execution of multiple potential motor programs. This is reflected in our data as poor discriminability on the single neuron level but robust discriminability on the subpopulation level.

These findings persisted even when we widened the ROC analysis window to encompass the entire $1 \mathrm{~s}$ illumination period, suggesting that distributed excitation is an important mechanism by which the indirect pathway achieves motor suppression. We found that the average inhibited cell subpopulation response also showed behavioral selectivity during the $1 \mathrm{~s}$ analysis window, even though it did not during the $100 \mathrm{~ms}$ analysis window. This is consistent with a model in which rapid excitation drives motor suppression, which may then be supported or otherwise finetuned by later onset inhibition in another subset of $\mathrm{SNr}$ cells. Similarly, Sano et al. (2013) recently found that immunotoxinmediated ablation of iMSNs reduced phasic $\mathrm{SNr}$ excitation in response to cortical stimulation, but had little effect on spontaneous $\mathrm{SNr}$ firing. These results further support the hypothesis that rapid excitation signals are an important means by which the indirect pathway circuit suppresses movement.

In summary, we have identified direct pathway-mediated inhibition and indirect pathway-mediated excitation as key determinants of locomotor activation and motor suppression in the SNr. These results are consistent both with theoretical predictions and with a recent study by Cui et al. (2013), which demonstrated, using optical recording techniques, that both direct and indirect pathway striatal MSNs increase firing at times before action selection. Together these data support the hypothesis that the direct pathway activates motor programs for execution while the indirect pathway exerts its effects by suppressing competing motor programs. In this scheme, the immobile states that we observe during widespread indirect pathway activation represent a state in which multiple potential motor programs are simultaneously suppressed.

In addition to identifying cell populations that strongly influence locomotor activation and motor suppression, our single trial behavioral analyses suggest that it is possible to decode $\mathrm{SNr}$ neural activity in real time to predict motor behavior. This approach will prove useful in refining our knowledge of normal basal ganglia circuit function and dysfunction in disorders such as Parkinson disease and Huntington disease. Real-time behavioral prediction may also aid in the development of therapeutic interventions such as adaptive deep brain stimulation, which uses measures of neural activity to improve stimulation efficacy (Little et al., 2013).

\section{References}

Albin RL, Young AB, Penney JB (1989) The functional anatomy of basal ganglia disorders. Trends Neurosci 12:366-375. CrossRef Medline

Anderson ME, Horak FB (1985) Influence of the globus pallidus on arm movements in monkeys. III. Timing of movement-related information. J Neurophysiol 54:433-448. Medline

Brotchie P, Iansek R, Horne MK (1991) Motor function of the monkey globus pallidus. 1. Neuronal discharge and parameters of movement. Brain 114:1667-1683. CrossRef Medline

Bryden DW, Johnson EE, Diao X, Roesch MR (2011) Impact of expected value on neural activity in rat substantia nigra pars reticulata. Eur J Neurosci 33:2308-2317. CrossRef Medline

Cui G, Jun SB, Jin X, Pham MD, Vogel SS, Lovinger DM, Costa RM (2013)
Concurrent activation of striatal direct and indirect pathways during action initiation. Nature 494:238-242. CrossRef Medline

DeLong MR (1990) Primate models of movement disorders of basal ganglia origin. Trends Neurosci 13:281-285. CrossRef Medline

Deniau JM, Kitai ST, Donoghue JP, Grofova I (1982) Neuronal interactions in the substantia nigra pars reticulata through axon collaterals of the projection neurons. An electrophysiological and morphological study. Exp Brain Res 47:105-113. Medline

Durieux PF, Schiffmann SN, de Kerchove d'Exaerde A (2012) Differential regulation of motor control and response to dopaminergic drugs by D1R and D2R neurons in distinct dorsal striatum subregions. EMBO J 31:640 653. Medline

Eagle DM, Wong JC, Allan ME, Mar AC, Theobald DE, Robbins TW (2011) Contrasting roles for dopamine D1 and D2 receptor subtypes in the dorsomedial striatum but not the nucleus accumbens core during behavioral inhibition in the stop-signal task in rats. J Neurosci 31:7349-7356. CrossRef Medline

Fan D, Rossi MA, Yin HH (2012) Mechanisms of action selection and timing in substantia nigra neurons. J Neurosci 32:5534-5548. CrossRef Medline

Georgopoulos AP, DeLong MR, Crutcher MD (1983) Relations between parameters of step-tracking movements and single cell discharge in the globus pallidus and subthalamic nucleus of the behaving monkey. J Neurosci 3:1586-1598. Medline

Gulley JM, Kuwajima M, Mayhill E, Rebec GV (1999) Behavior-related changes in the activity of substantia nigra pars reticulata neurons in freely moving rats. Brain Res 845:68-76. CrossRef Medline

Gulley JM, Kosobud AE, Rebec GV (2002) Behavior-related modulation of substantia nigra pars reticulata neurons in rats performing a conditioned reinforcement task. Neuroscience 111:337-349. CrossRef Medline

Hazrati LN, Parent A (1992) Convergence of subthalamic and striatal efferents at pallidal level in primates: an anterograde double-labeling study with biocytin and PHA-L. Brain Res 569:336-340. CrossRef Medline

Hikosaka O, Wurtz RH (1983) Visual and oculomotor functions of monkey substantia nigra pars reticulata. I. Relation of visual and auditory responses to saccades. J Neurophysiol 49:1230-1253. Medline

Isomura Y, Takekawa T, Harukuni R, Handa T, Aizawa H, Takada M, Fukai T (2013) Reward-modulated motor information in identified striatum neurons. J Neurosci 33:10209-10220. CrossRef Medline

Jahfari S, Waldorp L, van den Wildenberg WP, Scholte HS, Ridderinkhof KR, Forstmann BU (2011) Effective connectivity reveals important roles for both the hyperdirect (fronto-subthalamic) and the indirect (frontostriatal-pallidal) fronto-basal ganglia pathways during response inhibition. J Neurosci 31:6891-6899. CrossRef Medline

Jin X, Costa RM (2010) Start/stop signals emerge in nigrostriatal circuits during sequence learning. Nature 466:457-462. CrossRef Medline

Kravitz AV, Freeze BS, Parker PR, Kay K, Thwin MT, Deisseroth K, Kreitzer AC (2010) Regulation of parkinsonian motor behaviours by optogenetic control of basal ganglia circuitry. Nature 466:622-626. CrossRef Medline

Li CS, Yan P, Sinha R, Lee TW (2008) Subcortical processes of motor response inhibition during a stop signal task. Neuroimage 41:1352-1363. CrossRef Medline

Little S, Pogosyan A, Neal S, Zavala B, Zrinzo L, Hariz M, Foltynie T, Limousin P, Ashkan K, Fitzgerald J, Green AL, Aziz TZ, Brown P (2013) Adaptive deep brain stimulation in advanced Parkinson disease. Ann Neurol 74:449-457. Medline

Mailly P, Charpier S, Menetrey A, Deniau JM (2003) Three-dimensional organization of the recurrent axon collateral network of the substantia nigra pars reticulata neurons in the rat. J Neurosci 23:5247-5257. Medline

Meyer-Luehmann M, Thompson JF, Berridge KC, Aldridge JW (2002) Substantia nigra pars reticulata neurons code initiation of a serial pattern: implications for natural action sequences and sequential disorders. Eur J Neurosci 16:1599-1608. CrossRef Medline

Mink JW, Thach WT (1991) Basal ganglia motor control. II. Late pallidal timing relative to movement onset and inconsistent pallidal coding of movement parameters. J Neurophysiol 65:301-329. Medline

Mitchell SJ, Richardson RT, Baker FH, DeLong MR (1987) The primate globus pallidus: neuronal activity related to direction of movement. Exp Brain Res 68:491-505. Medline

Mushiake H, Strick PL (1995) Pallidal neuron activity during sequential arm movements. J Neurophysiol 74:2754-2758. Medline 
Nicolelis MA, Dimitrov D, Carmena JM, Crist R, Lehew G, Kralik JD, Wise SP (2003) Chronic, multisite, multielectrode recordings in macaque monkeys. Proc Natl Acad Sci U S A 100:11041-11046. CrossRef Medline

Parent A, Hazrati LN (1993) Anatomical aspects of information processing in primate basal ganglia. Trends Neurosci 16:111-116. CrossRef Medline

Quiroga RQ, Reddy L, Kreiman G, Koch C, Fried I (2005) Invariant visual representation by single neurons in the human brain. Nature 435:11021107. CrossRef Medline

Ryan LJ, Young SJ, Groves PM (1986) Substantia nigra stimulation evoked antidromic responses in rat neostriatum. Exp Brain Res 63:449-460. CrossRef Medline

Sagar SM, Sharp FR, Curran T (1988) Expression of c-fos protein in brain: metabolic mapping at the cellular level. Science 240:1328-1331. CrossRef Medline

Sano H, Chiken S, Hikida T, Kobayashi K, Nambu A (2013) Signals through the striatopallidal indirect pathway stop movements by phasic excitation in the substantia nigra. J Neurosci 33:7583-7594. CrossRef Medline

Sato M, Hikosaka O (2002) Role of primate substantia nigra pars reticulata in reward-oriented saccadic eye movement. J Neurosci 22:2363-2373. Medline
Schmidt R, Leventhal DK, Mallet N, Chen F, Berke JD (2013) Canceling actions involves a race between basal ganglia pathways. Nat Neurosci 16:1118-1124. CrossRef Medline

Sheng M, McFadden G, Greenberg ME (1990) Membrane depolarization and calcium induce c-fos transcription via phosphorylation of transcription factor CREB. Neuron 4:571-582. CrossRef Medline

Smith Y, Bevan MD, Shink E, Bolam JP (1998) Microcircuitry of the direct and indirect pathways of the basal ganglia. Neuroscience 86:353-387. CrossRef Medline

Sohal VS, Zhang F, Yizhar O, Deisseroth K (2009) Parvalbumin neurons and gamma rhythms enhance cortical circuit performance. Nature 459: 698-702. CrossRef Medline

Wenger KK, Musch KL, Mink JW (1999) Impaired reaching and grasping after focal inactivation of globus pallidus pars interna in the monkey. J Neurophysiol 82:2049-2060. Medline

Yizhar O, Fenno LE, Prigge M, Schneider F, Davidson TJ, O’Shea DJ, Sohal VS, Goshen I, Finkelstein J, Paz JT, Stehfest K, Fudim R, Ramakrishnan C, Huguenard JR, Hegemann P, Deisseroth K (2011) Neocortical excitation/inhibition balance in information processing and social dysfunction. Nature 477:171-178. CrossRef Medline 